\title{
PROPRIEDADES E CLASSIFICAÇÃO DA MADEIRA APLICADAS À SELEÇÃO DE GENÓTIPOS DE Eucalyptus ${ }^{1}$
}

\author{
Ronaldo Pereira Caixeta ${ }^{2}$, Paulo Fernando Trugilho³, Sebastião Carlos da Silva Rosado³ e José Tarcísio Lima²
}

\begin{abstract}
RESUMO - Os objetivos do presente estudo foram classificar e selecionar genótipos superiores de eucalipto por meio das propriedades de sua madeira, utilizando como ferramenta a análise de agrupamento, método de otimização de Tocher, com o intuito de obter múltiplos produtos. Foram utilizados 44 genótipos de eucaliptos, adaptados para as condições ambientais do noroeste de Minas Gerais. Para classificação dos materiais foram utilizadas características físicas, de resistência mecânica e químicas da madeira. Os resultados da análise de agrupamento classificaram os 44 genótipos em 11 grupos distintos, com alta variabilidade ou divergência entre si. De acordo com os valores médios das características apresentadas pelos grupos formados, foi possível definir novos clones de potencial, que certamente forneceram madeira de qualidade superior para atender a diversos usos. Os genótipos pertencentes aos grupos V, VI e VIII apresentam potencial favorável à produção de madeira serrada. Eles possibilitarão, também, a geração de híbridos artificiais a serem usados em futuros programas de melhoramento genético de Eucalyptus.
\end{abstract}

Palavras-chave: $\quad$ Propriedades da madeira, melhoramento florestal, Eucalyptus e análise de agrupamento.

\section{PROPERTIES AND CLASSIFICATION OF WOOD FOR SELECTING EucalyptuS GENOTYPES}

\begin{abstract}
The objective of this study was to classify and select superior genotypes of eucalypt based on the properties of its wood, utilizing cluster analysis, the Tocher's optimization method aiming to obtain various products. Forty-four genotypes adapted to the environmental conditions of northwestern Minas Gerais were used. Physical, mechanical resistance and chemical wood characteristics were used for the classification of the materials. Cluster analysis classified the genotypes in 11 different groups with high variability or divergence among them. According to the average values of the characteristics presented by the formed groups, it was possible to define new potential clones, which will certainly provide wood of superior quality. The genotypes from groups V, VI and VIII show potential for the production of sawed wood, also facilitating the generation of natural hybrids, which may be used in future programs of genetic improvement of Eucalyptus.
\end{abstract}

Key words: $\quad$ Wood properties, forest breeding, Eucalyptus, and cluster analysis.

\section{INTRODUÇÃO}

Atualmente, é consenso no setor florestal brasileiro que a madeira do gênero Eucalyptus poderá ser a principal matéria-prima para atender a diversos segmentos industriais, na produção de múltiplos produtos de madeira, e não somente para produção de carvão e celulose.
O Brasil tem um grande potencial para atender a esse novo mercado, pois possui a maior área plantada de eucalipto do mundo, com aproximadamente $2.920 .800 \mathrm{ha}$, sendo o Estado de Minas Gerais responsável por 53\% dessa área (Brandão, 1997). No entanto, o material genético oriundo dessas florestas, na sua maioria, não foi selecionado e conduzido com base nas características da madeira para obtenção de múltiplos produtos de

1 Recebido para publicação em 28.1.2001.

Aceito para publicação em 19.2.2003.

2 Mestre pela UfLA, Professor do Centro Universitário de Patos de Minas - UNIPAM, Patos de Minas-MG, $<$ ronaldocaixeta@acipatos.org.br>; ${ }^{3}$ Professores do Departamento de Ciências Florestais da - UFLA, 37200-000 Lavras-MG. 
qualidade. A maior parte dos estudos referentes às características da madeira concentrou-se apenas na densidade básica, no teor de lignina e nos elementos anatômicos.

Recentemente alguns pesquisadores no Brasil, como Trugilho (1995), Xavier (1996) e Garcia (1998), procuraram investigar as propriedades da madeira, por meio de técnicas que possibilitem a avaliação de um conjunto de características simultaneamente, para produção de celulose e carvão vegetal, ou seja, utilizando as técnicas de análise multivariada. Estas técnicas permitem aproveitar as correlações existentes entre as características, levando em consideração um nível de significância conhecido. No entanto, propriedades como resistência mecânica e retratibilidade da madeira, que são consideradas importantes para vários usos, não foram consideradas por nenhum desses pesquisadores, em suas análises.

Como o uso da madeira de eucalipto está se tornando cada vez mais diversificado, essas características devem ser incluídas em pesquisas que envolvam a seleção de material para atender aos usos estruturais e à produção de madeira serrada, para ser utilizada na fabricação de produtos sólidos.

Dessa forma, a classificação de genótipos superiores de eucaliptos para fins de seleção genética em um programa de melhoramento que possibilite sua aplicação na produção de múltiplos produtos deve ser feita com base nas propriedades da madeira, utilizando técnicas de avaliação conjunta.

Os objetivos do presente estudo foram classificar e selecionar genótipos superiores de eucalipto por meio das propriedades de sua madeira, utilizando como ferramenta a análise de agrupamento, método de otimização de Tocher, visando o aproveitamento de múltiplos produtos.

\section{MATERIAL E MÉTODOS}

\section{1. Amostragem}

Foram utilizados 44 genótipos superiores, com idades variando de 13 a 17 anos, obtidos em povoamentos de híbridos de Eucalyptus adaptados para as condições ambientais da região noroeste de Minas Gerais, no município de Vazante-MG. A latitude é de $17^{\circ} 36^{\prime} 09^{\prime \prime} \mathrm{S}$ e a longitude de $46^{\circ} 42^{\prime} 02^{\prime}$ " oeste de Greenwich, a uma altitude de $550 \mathrm{~m}$. O clima da área é do tipo Aw, tropical úmido de savana, com inverno seco e verão chuvoso,

R. Árvore, Viçosa-MG, v.27, n.1, p.43-51, 2003 segundo a classificação climática de Köppen. A temperatura média anual é de $24{ }^{\circ} \mathrm{C}$ e a precipitação média anual de $1.450 \mathrm{~mm}$. As árvores selecionadas foram abatidas, devidamente identificadas e amostradas. Na seleção fenotípica foram usadas as características de crescimento e tomou-se o cuidado de escolher somente as árvores que apresentassem o fuste mais linear e que possuíssem um bom estado fitossanitário.

Foram utilizadas as três primeiras toras de cada árvore com $3 \mathrm{~m}$ de comprimento, das quais foi retirada uma prancha central radial de 10 a $12 \mathrm{~cm}$ de espessura, conforme esquema mostrado na Figura 1. As pranchas foram usadas para determinação das características mecânicas, físicas e químicas da madeira.

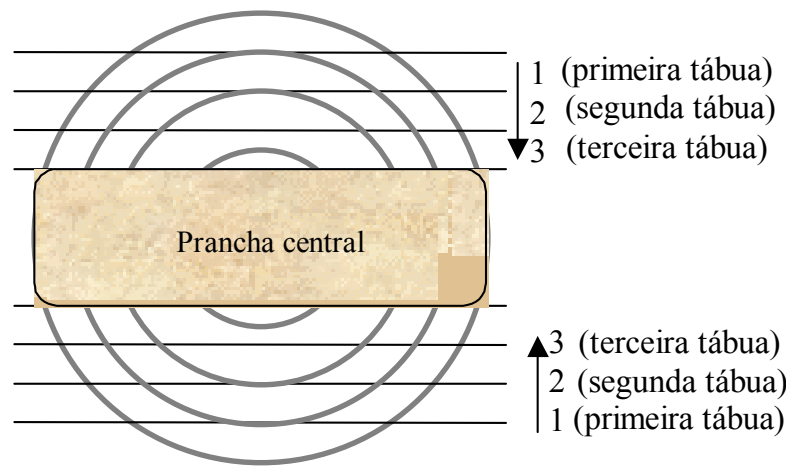

Figura 1 - Esquema ilustrativo da obtenção da prancha central utilizada.

Figure 1 - Scheme showing the cetral plank used.

\subsection{Características de Resistência Mecânica}

Foram realizados os seguintes ensaios para determinar a resistência da madeira: i) compressão paralela às fibras e módulo de elasticidade; ii) flexão estática, representada pelo módulo de ruptura (MDR) e pelo módulo de elasticidade; e iii) dureza janka. Os testes foram realizados conforme a norma da AMERICAN SOCIETY FOR TESTING AND MATERIALS (1997) D 143 - 94. No Quadro 1 estão as dimensões dos corposde-prova utilizados e a velocidade do ensaio.

\subsection{Características Físicas}

Foram determinadas as densidades básica, aparente e seca da madeira, de acordo com o método de imersão 
descrito por Vital (1984). O volume saturado foi determinado, utilizando a água e os volumes aparente e seco; o mercúrio foi empregado como líquido deslocado.

\begin{tabular}{|c|c|c|}
\hline Ensaio & $\begin{array}{c}\text { Dimensão do } \\
\text { Corpo-de-Prova } \\
(\mathrm{mm})\end{array}$ & $\begin{array}{c}\text { Velocidade do } \\
\text { Ensaio } \\
(\mathrm{mm} / \mathrm{min})\end{array}$ \\
\hline Compressão paralela às fibras & $50 \times 50 \times 200$ & 0,566 \\
\hline Flexão estática & $25 \times 25 \times 410$ & 1,300 \\
\hline Dureza janka & $50 \times 50 \times 150$ & 6,000 \\
\hline
\end{tabular}

Considerou-se a densidade aparente como sendo a relação entre a massa do corpo-de-prova seco em estufa e seu volume quando seco ao ar, ou seja, no equilíbrio higroscópio.

Para determinação das contrações da madeira foi utilizada a norma norte-americana AMERICAN SOCIETY FOR TESTING AND MATERIALS (1997) D 143 - 94, estabelecendo que as dimensões lineares sejam tomadas diretamente no corpo-de-prova, o que simplifica a medição, devendo ser ressaltado que as suas dimensões foram de 2,5 x 2,5 x 10,0 cm, livres de defeitos. A contração volumétrica foi determinada em função da relação entre diferentes volumes, tendo sido empregado o método de imersão para determinar tanto o volume verde quanto o absolutamente seco. As medições das dimensões lineares foram feitas com um paquímetro digital, com precisão de 0,01 mm. Estes corpos-de-prova foram usados na determinação das densidades.

\subsection{Características Químicas}

As análises químicas foram feitas para determinar o teor de lignina, de extrativos totais, de cinzas e de holocelulose e a solubilidade da madeira na água fria e quente. Para cada um deles foi utilizado um procedimento, conforme se segue:

- o teor de lignina (Klason) foi determinado de acordo com o procedimento descrito por Gomide e Demuner (1986); o teor de lignina solúvel em ácido sulfúrico foi determinado por meio da espectrofotometria, tendo sido utilizada a equação descrita por Goldschimid (1971); a lignina total foi tomada como sendo a soma das ligninas solúvel e insolúvel;

- o teor de extrativos totais foi obtido por meio da norma da ASSOCIAÇÃO BRASILEIRA TÉCNICA DE CELULOSE E PAPEL (1974) M 3/69;

- o teor de cinzas na madeira foi determinado de acordo com a norma ASSOCIAÇÃO BRASILEIRA TÉCNICA DE CELULOSE E PAPEL (1974) M 11/77;

- a solubilidade da madeira em água, fria e quente, foi determinada pela norma ASSOCIAÇÃO BRASILEIRA TÉCNICA DE CELULOSE E PAPEL (1974) M 4/68; e

- o teor de holocelulose foi obtido por diferença, por meio de análise somativa.

\section{5. Análise de Agrupamento ("Cluster Analysis")}

A análise de agrupamento foi utilizada para classificar os diversos indivíduos estudados em conjuntos de árvores com grandes semelhanças entre si. Esta análise reúne procedimentos que permitem classificar I populações em grupos, com base na medida de p características, de modo que exista homogeneidade dentro e heterogeneidade entre grupos (Marriott, 1974; Johnson \& Wichern, 1992).

O procedimento inicial é a conversão da matriz ${ }_{\mathrm{I}} \mathrm{Y}_{\mathrm{p}}=\left[\overline{\mathrm{Y}}_{\mathrm{ir}}\right]$ de médias amostrais em uma matriz ${ }_{\mathrm{I}} \mathrm{d}_{\mathrm{I}}=\left[\mathrm{d}_{\mathrm{ii}}\right]$ de dissimilaridade entre pares de genótipos (Johnson \& Wichern, 1992).

As análises de agrupamento foram conduzidas, adotando-se a distância euclidiana padronizada,

$\mathrm{dii}^{\prime}=\left[\frac{1}{\mathrm{n}} \sum_{\mathrm{j}}\left(\mathrm{X}_{\mathrm{ij}}-\mathrm{X}_{\mathrm{i}^{\prime} \mathrm{j}^{\prime}}\right)^{2}\right]^{1 / 2}$, em que

$X_{i j}=X_{i j} / S\left(X_{J}\right)$, como medida de dissimilaridade.

Foi empregado o método de otimização de Tocher, que é indicado para estudar o grau de semelhança existente entre genótipos, sendo utilizado na formação dos conjuntos de genótipos com maior semelhança entre si. $\mathrm{O}$ critério adotado pelo método de Tocher é que a média dos valores das distâncias intragrupos deve ser menor que as distâncias intergrupos (CRUZ, 1990).

R. Árvore, Viçosa-MG, v.27, n.1, p.43-51, 2003 
A distância média intragrupos é dada por:

$$
\mathrm{d}_{\mathrm{g}}=\sum_{\mathrm{i}, \mathrm{i}^{\prime}=1}^{\mathrm{I}} \frac{\mathrm{d}_{\mathrm{ii}}}{\mathrm{C}_{\mathrm{n}}^{2}}
$$

em que

$\mathrm{d}_{\mathrm{g}}=$ distância média dentro do grupo I;

$\sum_{\mathrm{i}, \mathrm{i}^{\prime}=1}^{\mathrm{I}} \mathrm{d}_{\mathrm{ii}}=$ soma de todas as possíveis combinações

dos $\mathbf{n}$ genótipos incluídos no grupo; e

$\mathrm{c}_{\mathrm{n}}^{2}=$ número de combinações possíveis.

A distância média intergrupos é dada por:

$$
\mathrm{d}_{\mathrm{g}, \mathrm{h}}=\sum_{\mathrm{i}, \mathrm{j}=1}^{\mathrm{I}} \frac{\mathrm{d}_{\mathrm{i} i^{\prime}}}{\mathrm{n}_{\mathrm{g}} \mathrm{n}_{\mathrm{h}}}
$$

em que

$\mathrm{d}_{\mathrm{g}, \mathrm{h}}=$ distância média entre os grupos $\mathbf{g}$ e $\mathbf{h}$;

$\sum_{i, j=1}^{I} d_{i i '}=$ soma de todas as possíveis combinações dos $\mathbf{n}_{\mathrm{g}} \times \mathbf{n}_{\mathbf{h}}$ genótipos incluídos nos grupos $\mathbf{g}$ e $\mathbf{h}$, respectivamente; $\mathrm{e}$

$\mathbf{n}_{\mathrm{g}} \times \mathbf{n}_{\mathrm{h}}=$ número total de combinações possíveis.

\section{RESULTADOS E DISCUSSÃO}

Os resultados relativos às médias e aos coeficientes de variação das características de resistência mecânica, física e química determinadas para os 44 genótipos encontram-se, respectivamente, nos Quadros 2, 3 e 4.

\section{1. Propriedades de Resistência Mecânica}

A resistência média à compressão paralela às fibras foi de $615 \mathrm{kgf} / \mathrm{cm}^{2}$, com módulo de elasticidade de $101.649 \mathrm{kgf} / \mathrm{cm}^{2}$. A flexão estática, representada pelo módulo de ruptura (MDR) médio, foi de $1.234 \mathrm{kgf} / \mathrm{cm}^{2} \mathrm{e}$ o módulo de elasticidade de $191.843 \mathrm{kgf} / \mathrm{cm}^{2}$. A dureza janka média determinada foi de $834 \mathrm{kgf}$.

Esses resultados foram superiores aos encontrados por Lima (1999), estudando clones de Eucalyptus grandis aos 8 anos de idade, em posição similar. Provavelmente, este fato está relacionado a diferenças genéticas, idade e ambiente, existentes entre os materiais.
Os genótipos pesquisados apresentaram resistência de média à alta, de acordo com a classificação proposta pela Sudam (1981), demonstrando assim a possibilidade de usos desses materiais pelos segmentos construção cívil e moveleiro.

Quadro 2 - Valores m édios das características de resistên cia m ecân ica em kgf/cm ${ }^{2}$ para os genótipos avaliados

rable 2 - Average values of the $m$ echanical resistance

\begin{tabular}{|c|c|c|c|c|c|}
\hline \multirow[t]{2}{*}{ Génotipo } & \multicolumn{2}{|c|}{$\begin{array}{c}\text { Compressão Paralela } \\
\text { às Fibras }\end{array}$} & \multicolumn{2}{|c|}{ Flexão Estática } & \multirow{2}{*}{$\begin{array}{l}\text { Dureza } \\
\text { Janka }\end{array}$} \\
\hline & $\mathrm{RC}$ & MOE & MDR & $\mathrm{MOE}$ & \\
\hline 1 & 567 & 95.730 & 1.307 & 176.052 & 1.148 \\
\hline 2 & 643 & 107.793 & 1.168 & 201.982 & 723 \\
\hline 3 & 607 & 104.486 & 1.138 & 200.252 & 634 \\
\hline 4 & 604 & 101.050 & 1.070 & 177.871 & 615 \\
\hline 5 & 500 & 85.323 & 988 & 145.605 & 751 \\
\hline 6 & 623 & 108.425 & 1.279 & 192.777 & 863 \\
\hline 7 & 687 & 112.601 & 1.419 & 242.593 & 1.100 \\
\hline 8 & 577 & 94.345 & 1.150 & 178.006 & 697 \\
\hline 9 & 575 & 94.825 & 1.037 & 173.314 & 566 \\
\hline 10 & 573 & 93.724 & 1.097 & 166.989 & 824 \\
\hline 11 & 544 & 92.092 & 1.222 & 172.973 & 635 \\
\hline 12 & 587 & 101.829 & 1.206 & 195.133 & 900 \\
\hline 13 & 596 & 100.616 & 1.216 & 191.765 & 754 \\
\hline 14 & 656 & 105.464 & 1.273 & 198.834 & 967 \\
\hline 15 & 544 & 90.400 & 1.014 & 141.986 & 910 \\
\hline 16 & 631 & 96.877 & 1.275 & 182.938 & 736 \\
\hline 17 & 700 & 110.186 & 1.459 & 218.321 & 991 \\
\hline 18 & 704 & 108.242 & 1.315 & 235.140 & 818 \\
\hline 19 & 612 & 100.379 & 1.174 & 169.915 & 776 \\
\hline 20 & 669 & 111.965 & 1.182 & 192.093 & 992 \\
\hline 21 & 651 & 107.469 & 1.309 & 199.418 & 875 \\
\hline 22 & 510 & 86.806 & 1.084 & 152.819 & 586 \\
\hline 23 & 567 & 92.450 & 1.145 & 172.308 & 703 \\
\hline 24 & 644 & 102.758 & 1.273 & 191.497 & 1.000 \\
\hline 25 & 568 & 94.715 & 1.147 & 175.960 & 596 \\
\hline 26 & 669 & 111.801 & 1.275 & 194.940 & 1.114 \\
\hline 27 & 659 & 114.681 & 1.313 & 203.589 & 987 \\
\hline 28 & 591 & 96.474 & 1.197 & 174.105 & 727 \\
\hline 29 & 581 & 93.695 & 1.179 & 163.680 & 943 \\
\hline 30 & 576 & 95.766 & 1.192 & 181.453 & 565 \\
\hline 31 & 653 & 105.276 & 1.391 & 209.910 & 853 \\
\hline 32 & 656 & 111.355 & 1.357 & 204.807 & 1.011 \\
\hline 33 & 680 & 111.367 & 1.393 & 244.885 & 856 \\
\hline 34 & 670 & 109.833 & 1.394 & 217.875 & 980 \\
\hline 35 & 613 & 102.516 & 1.157 & 189.979 & 713 \\
\hline 36 & 644 & 102.341 & 1.301 & 200.333 & 891 \\
\hline 37 & 564 & 93.752 & 1.146 & 172.775 & 731 \\
\hline 38 & 578 & 97.033 & 1.278 & 199.301 & 738 \\
\hline 39 & 655 & 107.002 & 1.438 & 223.810 & 968 \\
\hline 40 & 661 & 105.286 & 1.362 & 205.992 & 1.044 \\
\hline 41 & 690 & 114.417 & 1.431 & 242.748 & 839 \\
\hline 42 & 596 & 98.550 & 1.148 & 182.783 & 785 \\
\hline 43 & 590 & 100.614 & 1.104 & 192.140 & 778 \\
\hline 44 & 611 & 102.246 & 1.274 & 189.458 & 1.001 \\
\hline Média & 615 & 101.694 & 1.234 & 191.843 & 834 \\
\hline $\mathrm{CV}(\%)$ & 8,18 & 7,52 & 9,71 & 12,44 & 18,76 \\
\hline
\end{tabular}
characteristics in $\mathrm{kgf} / \mathrm{cm} 2$ for the genotypes.

$\mathrm{RC}=$ resistência à compressão $\left(\mathrm{kgf} / \mathrm{cm}^{2}\right), \mathrm{MOE}=$ módulo de elasticidade $\left(\mathrm{kgf} / \mathrm{cm}^{2}\right), \mathrm{MDR}=$ módulo de ruptura $\left(\mathrm{kgf} / \mathrm{cm}^{2}\right)$, dureza janka (kgf) e $\mathrm{CV}(\%)=$ coeficiente de variação em porcentagem. 
Q uadro 3 - Valores m édios das características físicas para os genótipos analisados

Table 3 - A verage values of the physical characteristics for the genotypes ana lyzed

\begin{tabular}{|c|c|c|c|c|c|c|c|c|}
\hline Genótipo & CT & CR & $\mathrm{CL}$ & $\mathrm{CV}$ & $\mathrm{CT} / \mathrm{CR}$ & DS & DB & $\mathrm{DA}$ \\
\hline 1 & 8,98 & 5,22 & 0,19 & 12,53 & 1,74 & 0,813 & 0,711 & 0,767 \\
\hline 2 & 10,01 & 7,35 & 0,33 & 15,76 & 1,38 & 0,765 & 0,644 & 0,715 \\
\hline 3 & 10,13 & 6,42 & 0,25 & 14,88 & 1,59 & 0,702 & 0,597 & 0,662 \\
\hline 4 & 9,76 & 6,33 & 0,28 & 15,74 & 1,57 & 0,661 & 0,557 & 0,627 \\
\hline 5 & 8,82 & 5,21 & 0,31 & 13,67 & 1,72 & 0,679 & 0,586 & 0,643 \\
\hline 6 & 9,75 & 7,25 & 0,21 & 16,20 & 1,36 & 0,775 & 0,650 & 0,728 \\
\hline 7 & 11,21 & 7,31 & 0,16 & 17,11 & 1,57 & 0,882 & 0,731 & 0,817 \\
\hline 8 & 11,17 & 6,72 & 0,30 & 16,28 & 1,72 & 0,694 & 0,582 & 0,655 \\
\hline 9 & 10,87 & 7,45 & 0,34 & 17,15 & 1,48 & 0,679 & 0,562 & 0,639 \\
\hline 10 & 9,22 & 5,02 & 0,29 & 13,34 & 1,91 & 0,718 & 0,622 & 0,677 \\
\hline 11 & 7,59 & 5,88 & 0,30 & 12,17 & 1,33 & 0,723 & 0,635 & 0,682 \\
\hline 12 & 9,55 & 6,34 & 0,28 & 15,57 & 1,53 & 0,759 & 0,641 & 0,713 \\
\hline 13 & 9,11 & 5,96 & 0,36 & 15,33 & 1,55 & 0,706 & 0,597 & 0,664 \\
\hline 14 & 8,60 & 6,35 & 0,20 & 15,40 & 1,37 & 0,786 & 0,665 & 0,739 \\
\hline 15 & 8,16 & 5,77 & 0,32 & 14,77 & 1,43 & 0,778 & 0,664 & 0,733 \\
\hline 16 & 8,94 & 5,98 & 0,25 & 15,07 & 1,51 & 0,729 & 0,619 & 0,685 \\
\hline 17 & 10,01 & 6,92 & 0,28 & 17,58 & 1,45 & 0,805 & 0,663 & 0,748 \\
\hline 18 & 10,51 & 7,43 & 0,28 & 18,60 & 1,43 & 0,778 & 0,633 & 0,724 \\
\hline 19 & 9,83 & 6,72 & 0,22 & 16,80 & 1,48 & 0,715 & 0,595 & 0,673 \\
\hline 20 & 11,50 & 8,28 & 0,29 & 20,15 & 1,41 & 0,807 & 0,645 & 0,757 \\
\hline 21 & 10,80 & 6,59 & 0,19 & 18,27 & 1,68 & 0,766 & 0,626 & 0,713 \\
\hline 22 & 8,88 & 5,49 & 0,26 & 15,38 & 1,71 & 0,643 & 0,544 & 0,602 \\
\hline 23 & 9,14 & 6,58 & 0,28 & 15,79 & 1,43 & 0,730 & 0,615 & 0,689 \\
\hline 24 & 9,74 & 6,84 & 0,27 & 17,03 & 1,45 & 0,813 & 0,675 & 0,759 \\
\hline 25 & 9,26 & 5,67 & 0,25 & 15,55 & 1,65 & 0,673 & 0,569 & 0,632 \\
\hline 26 & 10,89 & 7,19 & 0,27 & 17,39 & 1,52 & 0,810 & 0,669 & 0,759 \\
\hline 27 & 9,74 & 6,17 & 0,26 & 15,58 & 1,60 & 0,820 & 0,692 & 0,769 \\
\hline 28 & 9,44 & 6,44 & 0,24 & 16,08 & 1,47 & 0,686 & 0,576 & 0,648 \\
\hline 29 & 9,01 & 6,28 & 0,25 & 15,50 & 1,45 & 0,794 & 0,672 & 0,745 \\
\hline 30 & 9,78 & 5,65 & 0,21 & 16,06 & 1,79 & 0,684 & 0,576 & 0,653 \\
\hline 31 & 9,13 & 7,09 & 0,26 & 16,18 & 1,30 & 0,785 & 0,658 & 0,739 \\
\hline 32 & 10,86 & 6,72 & 0,26 & 17,77 & 1,63 & 0,829 & 0,681 & 0,779 \\
\hline 33 & 10,89 & 6,88 & 0,39 & 17,88 & 1,64 & 0,779 & 0,640 & 0,729 \\
\hline 34 & 9,94 & 6,15 & 0,19 & 16,75 & 1,64 & 0,819 & 0,682 & 0,771 \\
\hline 35 & 10,00 & 5,87 & 0,27 & 16,37 & 1,77 & 0,673 & 0,562 & 0,636 \\
\hline 36 & 10,68 & 6,06 & 0,44 & 17,31 & 1,78 & 0,786 & 0,650 & 0,742 \\
\hline 37 & 11,19 & 6,31 & 0,29 & 18,42 & 1,81 & 0,670 & 0,546 & 0,635 \\
\hline 38 & 9,00 & 6,25 & 0,28 & 16,14 & 1,46 & 0,725 & 0,608 & 0,684 \\
\hline 39 & 11,75 & 6,86 & 0,34 & 18,98 & 1,74 & 0,824 & 0,667 & 0,778 \\
\hline 40 & 9,00 & 6,25 & 0,23 & 16,84 & 1,47 & 0,788 & 0,655 & 0,741 \\
\hline 41 & 10,13 & 5,84 & 0,23 & 16,77 & 1,75 & 0,762 & 0,634 & 0,717 \\
\hline 42 & 8,63 & 5,81 & 0,29 & 15,56 & 1,50 & 0,710 & 0,600 & 0,672 \\
\hline 43 & 9,07 & 5,93 & 0,31 & 15,12 & 1,56 & 0,768 & 0,651 & 0,725 \\
\hline 44 & 9,69 & 6,07 & 0,27 & 16,45 & 1,62 & 0,765 & 0,636 & 0,723 \\
\hline Média & 9,78 & 6,38 & 0,27 & 16,21 & 1,57 & 0,751 & 0,629 & 0,707 \\
\hline $\mathrm{CV}(\%)$ & 9,62 & 10,61 & 20,17 & 9,75 & 9,46 & 7,534 & 7,167 & 7,225 \\
\hline
\end{tabular}

CT, CR, CL e CV referem-se, respectivamente, às contrações tangencial, radial, longitudinal e volumétrica; $\mathrm{CT} / \mathrm{CR}=$ coeficiente de anisotropia; DS, DB e DA refere-se, respectivamente, às densidades seca, básica e aparente; e $\operatorname{CV}(\%)=$ coeficiente de variação em porcentagem.

\subsection{Propriedades Físicas}

Os resultados médios encontrados para as densidades e as contrações apresentaram grande amplitude de variação entre os indivíduos, o que proporciona uma seleção para usos diversificados. O coeficiente de
Quadro 4 - Valores m édios das características quím icas para os genótipos avaliados

Table 4 - A verage values of the chem ical characteristics for the genotypes evaluated

\begin{tabular}{|c|c|c|c|c|c|c|}
\hline Genótipo & $\mathrm{CZ}$ & EAF & EAQ & ET & LIG & HOLO \\
\hline 1 & 0,078 & 4,59 & 6,33 & 7,61 & 32,51 & 59,80 \\
\hline 2 & 0,168 & 4,74 & 6,33 & 7,73 & 27,72 & 64,39 \\
\hline 3 & 0,202 & 4,15 & 5,05 & 6,42 & 30,52 & 62,86 \\
\hline 4 & 0,122 & 3,48 & 4,56 & 6,27 & 24,24 & 69,37 \\
\hline 5 & 0,113 & 2,81 & 4,16 & 5,18 & 27,20 & 67,51 \\
\hline 6 & 0,142 & 4,18 & 4,92 & 5,58 & 29,10 & 65,18 \\
\hline 7 & 0,103 & 2,12 & 2,70 & 3,23 & 25,90 & 70,77 \\
\hline 8 & 0,228 & 4,32 & 5,66 & 6,71 & 29,25 & 63,82 \\
\hline 9 & 0,257 & 3,30 & 4,38 & 4,72 & 26,96 & 68,07 \\
\hline 10 & 0,123 & 5,31 & 6,41 & 7,70 & 29,07 & 63,11 \\
\hline 11 & 0,172 & 3,80 & 4,45 & 5,50 & 32,14 & 62,20 \\
\hline 12 & 0,103 & 2,76 & 3,38 & 5,03 & 27,20 & 67,67 \\
\hline 13 & 0,128 & 2,63 & 3,35 & 4,28 & 30,77 & 64,82 \\
\hline 14 & 0,083 & 4,29 & 5,65 & 6,66 & 30,53 & 62,73 \\
\hline 15 & 0,113 & 4,79 & 6,55 & 7,41 & 31,60 & 60,87 \\
\hline 16 & 0,120 & 5,06 & 6,10 & 6,83 & 29,17 & 63,89 \\
\hline 17 & 0,110 & 2,65 & 3,17 & 4,42 & 26,79 & 68,69 \\
\hline 18 & 0,143 & 1,70 & 2,68 & 3,64 & 26,33 & 69,89 \\
\hline 19 & 0,085 & 4,25 & 5,43 & 6,36 & 31,08 & 62,48 \\
\hline 20 & 0,148 & 3,19 & 4,01 & 5,02 & 28,83 & 66,00 \\
\hline 21 & 0,117 & 3,60 & 4,63 & 5,38 & 26,30 & 68,20 \\
\hline 22 & 0,130 & 3,96 & 4,82 & 5,86 & 31,37 & 62,64 \\
\hline 23 & 0,080 & 5,50 & 6,90 & 8,00 & 32,31 & 59,62 \\
\hline 24 & 0,150 & 4,16 & 4,91 & 6,15 & 30,26 & 63,44 \\
\hline 25 & 0,173 & 2,77 & 3,79 & 4,94 & 28,73 & 66,16 \\
\hline 26 & 0,105 & 3,83 & 4,60 & 5,97 & 29,95 & 63,97 \\
\hline 27 & 0,185 & 3,64 & 4,64 & 5,81 & 33,06 & 60,94 \\
\hline 28 & 0,113 & 3,93 & 4,49 & 6,06 & 25,97 & 67,86 \\
\hline 29 & 0,057 & 5,26 & 6,65 & 8,76 & 27,90 & 63,29 \\
\hline 30 & 0,065 & 6,23 & 7,29 & 9,28 & 31,32 & 59,34 \\
\hline 31 & 0,115 & 4,82 & 5,94 & 6,56 & 27,22 & 66,11 \\
\hline 32 & 0,085 & 5,76 & 7,62 & 8,92 & 27,52 & 63,48 \\
\hline 33 & 0,120 & 4,75 & 5,48 & 6,10 & 23,77 & 70,01 \\
\hline 34 & 0,057 & 3,72 & 4,79 & 6,30 & 28,84 & 64,80 \\
\hline 35 & 0,117 & 3,49 & 3,81 & 5,24 & 28,61 & 66,03 \\
\hline 36 & 0,112 & 4,98 & 5,57 & 7,07 & 31,39 & 61,43 \\
\hline 37 & 0,132 & 3,50 & 4,35 & 5,94 & 27,99 & 65,95 \\
\hline 38 & 0,070 & 4,06 & 4,72 & 5,70 & 28,25 & 65,99 \\
\hline 39 & 0,100 & 4,90 & 6,11 & 8,40 & 30,31 & 61,20 \\
\hline 40 & 0,088 & 4,29 & 6,38 & 6,55 & 31,05 & 62,31 \\
\hline 41 & 0,083 & 3,04 & 3,59 & 5,08 & 27,80 & 67,05 \\
\hline 42 & 0,118 & 4,59 & 5,05 & 6,80 & 28,82 & 64,26 \\
\hline 43 & 0,113 & 4,77 & 5,72 & 7,11 & 29,49 & 63,29 \\
\hline 44 & 0,097 & 6,00 & 7,94 & 9,31 & 29,17 & 61,42 \\
\hline Média & 0,121 & 4,08 & 5,11 & 6,31 & 28,96 & 64,61 \\
\hline $\mathrm{CV}(\%)$ & 35,033 & 24,957 & 24,931 & 22,424 & 7,525 & 4,585 \\
\hline
\end{tabular}

$\mathrm{CZ}=$ teor de cinzas $(\%), \mathrm{EAF}=$ extrativos solúveis em água fria $(\%)$, EAQ $=$ extrativos solúveis em água quente $(\%), \mathrm{ET}=$ teor de extrativos totais $(\%), \mathrm{LIG}=$ teor de lignina total $(\%)$, HOLO = teor de hocelulose $(\%)$ e $\mathrm{CV}(\%)=$ coeficiente de variação em porcentagem.

variação ficou abaixo de $10 \%$, com exceção das contrações radial e longitudinal, que apresentaram, respectivamente, 10,61 e $20,17 \%$. Estes resultados estão todos de acordo com os citados na literatura.

De modo geral, os genótipos pesquisados apresentaram contrações elevadas, de acordo a classificação 
propostas pela Sudam (1981). No entanto, devido à grande amplitude de variação existente entre os materiais, é possível selecionar genótipos com porcentagem de contração volumétrica total inferior a $14 \%$.

Em relação à característica densidade, considerando a classificação da Sudam (1981), constatou-se que 42\% dos genótipos avaliados foram classificados como madeira de "peso" médio e os demais foram considerados como madeira pesada.

Esses resultados indicam a possibilidade de usos diversificados para tais materiais, uma vez que a densidade é considerada um dos indicadores básicos para a maioria das aplicações da madeira. Por exemplo, a madeira de densidade média poderá ser indicada para móveis, celulose e papel etc. A madeira de densidade superior poderá ser indicada para fins estruturais, como a fabricação de casas e pontes.

\subsection{Propriedades Químicas}

Quanto aos componentes químicos avaliados, verificou-se que todos resultados médios encontrados estão de acordo com a literatura. O coeficiente de variação foi maior que $20 \%$ para quase todas as características, com exceção do teor de lignina e holocelulose, os quais apresentaram, respectivamente, 7,52 e 4,58\% de variação em relação à média. Este resultado é considerado normal, tendo em vista que os genótipos avaliados apresentam grandes diferenças genéticas.

\subsection{Análises de Agrupamento}

Preliminarmente, foi realizado um teste para diagnóstico da multicolineariade ou dependência linear na matriz de correlação fenotípica, a fim de evitar o uso de variáveis redundantes. Após essa análise, foram identificadas e descartadas as variáveis que provocavam forte multicolinearidade (variáveis redundantes). A técnica utilizada na identificação e no descarte das variáveis consideradas redundantes foi baseada nos fatores de inflação da variância, magnitude dos autovalores e valor singular, contidas em Cruz (1990). As características com autovalores muito baixos podem ser descartadas, pois são consideradas de pouca importância ou redundantes.

As características que provocaram forte multicolinearidade foram o módulo de elasticidade à compressão paralela às fibras, o módulo de elasticidade à flexão

R. Árvore, Viçosa-MG, v.27, n.1, p.43-51, 2003 estática, a dureja janka, a contração tangencial, a contração longitudinal, a densidade seca, a densidade aparente, o teor de extrativos totais, os extrativos solúveis em água fria e o teor de holoceluloce, portanto estas características foram eliminadas da matriz de correlação.

Foram utilizadas as distâncias euclidianas padronizadas como medida de dissimilaridade e o método de otimização de Tocher para a análise de agrupamento, o qual proporcionou a formação de 11 grupos entre os genótipos estudados, conforme o Quadro 5.

Pelo Quadro 5 verifica-se que o grupo I foi formado com $50 \%$ dos genótipos. Os demais foram distribuídos em dez grupos distintos, o que permite a obtenção de genótipos diferenciados, quanto às características analisadas conjuntamente.

A variabilidade existente entre os genótipos é de vital importância, tanto para multiplicação clonal como para formação de pomar de sementes. A multiplicação clonal, visando produzir madeira com características desejáveis para usos específicos, pode ser realizada pela utilização do material presente no grupo considerado de melhor qualidade. No entanto, quando se deseja a instalação de pomar de sementes para proporcionar maior recombinação pelos cruzamentos entre os genitores divergentes, devem ser utilizados os genótipos provenientes de grupos diferentes, principalmente aqueles que apresentaram as maiores distâncias genéticas. As sementes originadas do pomar formado por estas árvores permitirão a condução de novos ciclos de seleção e poderão restabelecer a variabilidade genética nas populações de eucalipto para produção de múltiplos produtos de madeira.

No Quadro 6 estão as distâncias médias inter e intragrupos. Observa-se que os valores médios de distâncias dentro dos grupos foram menores que as distâncias entre grupos, o que confirma o critério estabelecido pela metodologia de Tocher.

Os resultados observados indicam que as maiores distâncias ocorreram entre os grupos IX e VI $(8,09)$, XI e VI $(8,05)$, XI e VII $(7,66)$ e XI e IV $(7,30)$. As menores distâncias foram encontradas entre os grupos I e X $(3,63)$, II e VIII $(3,65)$ e IV e VIII $(3,68)$. O grupo XI apresentou, de modo geral, os maiores valores de distância em relação aos demais.

No Quadro 7 estão os valores médios das características dentro de cada grupo formado. Os resultados demonstram haver grande variabilidade entre os grupos. 
Propriedades e Classificação da Madeira Aplicadas à ...

$Q$ uadro 5 - A grupam ento estabelecido pelo m étodo de Tocher de 44 genótipos de Eucalyptus

Table 5 - C lustering established by the Tocher's $m$ ethod of $44 \mathrm{E} u$ calyptus genotypes

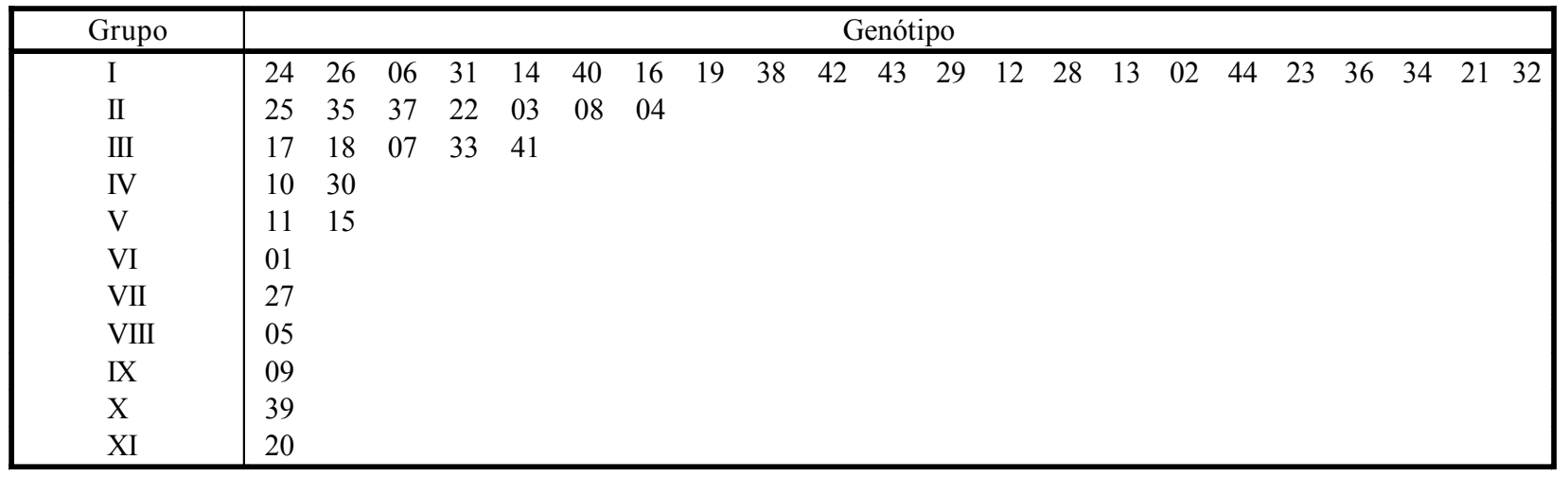

$Q$ u adro 6 - D istân cias m édias intragrupos, na diagonal, e intergrupos na diagonalabaixo

Table $6-A$ verage distance $w$ ith in groups, diagonally, and am ong groups in the diagonal below

\begin{tabular}{|l|c|c|c|c|c|c|c|c|c|c|c|}
\hline Grupo & I & II & III & IV & V & VI & VII & VIII & IX & X & XI \\
\hline I & $\mathbf{2 , 9 8}$ & & & & & & & & & & \\
II & 4,01 & $\mathbf{2 , 9 4}$ & & & & & & & & \\
III & 4,07 & 5,28 & $\mathbf{2 , 9 5}$ & & & & & & & \\
IV & 4,37 & 3,95 & 6,30 & $\mathbf{3 , 0 2}$ & & & & & & \\
V & 4,17 & 4,51 & 6,44 & 4,53 & $\mathbf{3 , 3 8}$ & & & & & \\
VI & 4,77 & 5,62 & 6,53 & 3,75 & 4,12 & - & & & & \\
VII & 3,83 & 4,61 & 4,72 & 5,08 & 4,24 & 4,32 & - & & & \\
VIII & 5,09 & 3,65 & 5,56 & 3,68 & 4,36 & 5,14 & 6,16 & - & & & \\
IX & 5,33 & 4,09 & 6,09 & 6,58 & 5,74 & 8,09 & 5,68 & 5,80 & - & & \\
X & 3,63 & 5,13 & 3,76 & 5,09 & 6,07 & 5,38 & 3,93 & 6,96 & 6,55 & - & \\
XI & 4,59 & 5,54 & 4,14 & 7,30 & 6,60 & 8,05 & 5,19 & 7,66 & 4,61 & 4,40 & - \\
\hline
\end{tabular}

$Q$ uadro 7 - V alores m édios das características dentro de cada agrupam ento form ado de genótipos de $E$ ucalyptus $\mathbf{T}$ able 7 - A verage values of the characteristics $w$ ith in each group of $\boldsymbol{E} \mathbf{u}$ ca lyptu senotypes

\begin{tabular}{|l|c|c|c|c|c|c|c|c|c|}
\hline \multirow{2}{*}{ Grupo } & \multicolumn{10}{|c|}{ Característica } \\
\cline { 2 - 10 } & RC & MDR & CR & CV & CT/CR & DB & CINZA & EAQ & LIG \\
\hline I & 623 & 1.254 & 6,46 & 16,29 & 1,51 & 0,639 & 0,11 & 5,50 & 29,09 \\
II & 577 & 1.127 & 6,12 & 16,09 & 1,69 & 0,565 & 0,16 & 4,58 & 28,67 \\
III & 692 & 1.404 & 6,88 & 17,59 & 1,57 & 0,660 & 0,11 & 3,52 & 26,12 \\
IV & 575 & 1.145 & 5,34 & 14,70 & 1,85 & 0,599 & 0,10 & 6,85 & 30,20 \\
V & 544 & 1.118 & 5,83 & 13,47 & 1,38 & 0,650 & 0,14 & 5,50 & 31,87 \\
VI & 567 & 1.307 & 5,22 & 12,53 & 1,74 & 0,711 & 0,08 & 6,33 & 32,51 \\
VII & 659 & 1.313 & 6,17 & 15,58 & 1,60 & 0,692 & 0,19 & 4,64 & 33,06 \\
VIII & 500 & 988 & 5,21 & 13,67 & 1,72 & 0,586 & 0,11 & 4,16 & 27,20 \\
IX & 575 & 1.037 & 7,45 & 17,15 & 1,48 & 0,562 & 0,26 & 4,38 & 26,96 \\
X & 655 & 1.438 & 6,86 & 18,98 & 1,74 & 0,667 & 0,10 & 6,11 & 30,31 \\
XI & 669 & 1.182 & 8,28 & 20,15 & 1,41 & 0,645 & 0,15 & 4,01 & 28,83 \\
\hline
\end{tabular}

$\mathrm{RC}=$ resistência à compressão paralela às fibras $\left(\mathrm{kgf} / \mathrm{cm}^{2}\right), \mathrm{MDR}=$ módulo de ruptura à flexão estática, $\mathrm{CR}$ e $\mathrm{CV}=$ contração radial $(\%)$, volumétrica, $\mathrm{CT} / \mathrm{CR}=$ coeficiente de anisotropia; $\mathrm{DB}=$ densidade básica $\left(\mathrm{g} / \mathrm{cm}^{3}\right)$, cinza $=$ teor de cinzas $(\%)$, EAQ = extrativos solúveis em água quente em (\%) e LIG = teor de lignina total (\%). 
Pelos resultados apresentados no Quadro 7, verificou-se que os grupos V, VI e VIII apresentaram os menores valores médios de contração volumétrica, associados a baixos valores de contração radial e coeficientes de anisotropia, o que indica a transformação desta madeira em tábuas e posterior usinagem para atender ao setor moveleiro na produção de painéis, molduras e componentes para móveis. A resistência mecânica para estes grupos foi a menor em relação aos demais, portanto este material não é o mais indicado para uso estrutural. $\mathrm{O}$ grupo $\mathrm{V}$ apresentou a madeira de maior estabilidade dimensional.

Os grupos II e IX apresentaram características favoráveis para produção de celulose, pois possuem menores valores médios para a característica teor de lignina total e também por possuírem densidade básica considerada ideal para essa finalidade.

O grupo IV é caracterizado principalmente pelos altos valores médios em extrativos solúvel em água quente e lignina total. Desta forma, este grupo não é indicado para uso como a produção de celulose, em razão de provocar maior aumento no consumo de reagentes durante o processo de deslignificação da madeira. Entretanto, ele pode ser utilizado para produção de carvão vegetal.

Os grupos VII, X e XI foram formados por genótipos que apresentaram os maiores valores médios em resistência mecânica e densidade básica. Os grupos VII e X apresentaram valores elevados no teor de lignina total. Os genótipos pertencentes ao grupo VII oferecem alternativas de emprego como pisos e assoalhos, decks, esquadrias e estrutural para atender ao segmento imobiliário. Outro produto que poderá ser obtido com os genótipos, principalmente os dos grupos VII e X, é a produção de carvão vegetal de alta qualidade, devido ao elevado teor de lignina total.

O grupo I, onde está concentrada a maioria dos genótipos, apresentou valores médios intermediários para as características avaliadas, sendo o mais promissor para atender à maioria dos requisitos necessários para produção de diversos produtos, embora os ganhos para algumas características possam ser maximizados em outros, por meio da hibridação.

Os grupos III, IX, X e XI apresentaram os maiores valores médios para contração volumétrica, indicando assim possuírem madeira com alta tensão residual de crescimento, o que pode levar ao seu descarte na utilização para serraria.

R. Árvore, Viçosa-MG, v.27, n.1, p.43-51, 2003

\section{CONCLUSÕES}

As principais conclusões obtidas no presente estudo foram:

- Por meio da distância euclidiana padronizada e pelo método de otimização de Tocher foi possível definir 11 grupos de genótipos com características favoráveis para melhorar a qualidade dos produtos oriundos da matéria-prima madeira.

- No grupo I, 50\% dos genótipos apresentaram valores médios intermediários para todas as características avaliadas, o que indica que este grupo tem maiores possibilidades de usos da madeira.

- A contração volumétrica, associada aos baixos valores de contração radial e ao coeficiente de anisotropia, foi menor nos grupos V, VI e VIII, o que indica a superioridade de seus genótipos para uso como madeira para serraria.

- Os grupos II, III e IX apresentam características favoráveis para produção de celulose, pois possuem baixos valores médios para o teor de lignina total e também por apresentarem valores em densidade básica considerados ideais para este uso.

- Os grupos VII, X e XI foram formados por genótipos que apresentaram altos valores médios em resistência mecânica e densidade básica. Os genótipos pertencentes ao grupo VII oferecem alternativas de emprego como pisos e assoalhos, decks, esquadrias e estruturas para o segmento imobiliário. Outro produto que poderá ser obtido com os genótipos, principalmente dos grupos VII e X, é a produção de carvão vegetal de alta qualidade, devido ao elevado teor de lignina total.

- Os grupos III, IX, X e XI apresentaram os maiores valores médios para contração volumétrica, indicando assim possuírem madeira com alta tensão residual de crescimento, o que pode levar ao seu descarte na utilização para serraria.

- Os genótipos apresentaram grande variabilidade ou divergência, o que é interessante do ponto de vista do melhoramento florestal.

\section{REFERÊNCIAS BIBLIOGRÁFICAS}

AMERICAN SOCIETY FOR TESTING AND MATERIALS - ASTM. 1997. Annual book of ASTM. Denvers: 1997. 679 p. D 143-94. Standard methods of testing small, clear specimens of timber, p. 23-53. 
ASSOCIAÇÃO BRASILEIRA TÉCNICA DE CELULOSE E PAPEL-ABTCP. Normas técnicas ABCP. São Paulo: 1974. não paginado.

BRANDÃO, L.G. Desafio florestal brasileiro. Silvicultura, v. 17, n. 73 , p. 23-29, set./dez, 1997.

CRUZ, C. D.; REGAZZI, A. J. Modelos biométricos aplicados ao melhoramento genético. Viçosa, MG: UFV, 1994. $390 \mathrm{p}$.

CRUZ, C. D. Aplicação de algumas técnicas multivariadas no melhoramento de plantas. $1990.188 \mathrm{f}$ Tese (Doutorado em Genética e Melhoramento de Planta) Escola Superior de Agricultura "Luiz de Queiroz", Piracicaba, 1990.

GARCIA, S. L. R. Importância de características de crescimento, de qualidade da madeira e da polpa na diversidade genética de clones de eucalipto. 1998. $103 \mathrm{f}$. Dissertação (Mestrado em Ciência Florestal) - Universidade Federal de Viçosa, Viçosa-MG, 1998.

GOLDSCHIMID, O. Ultraviolet spectra. In: SARKANEN, K. V.; LUDWIG, C. H. Lignins: occurrence, formation, structure and reactions. New York: John Wiley \& Sons, 1971. p. 241-266.

GOMIDE, J. L.; DEMUNER, B. J. Determinação do teor de lignina em material lenhoso: método Klason modificado. O Papel, v. 47, n. 8, p. 36-38, 1986.
JOHNSON, R. A; WICHERN, D. W. Applied multivariate statistical analysis. New Jersey: Prentice-Hall, 1992. $607 \mathrm{p}$.

LIMA, J. T. Clonal variation in the solid wood properties of Eucalyptus. 1999. 276 f. Thesis (Ph.D.) - University of Wales, Bangor, 1999.

MARRIOTT, F. H. C. The interpretation of multiple observation. New York: Academic Press, 1974. 117 p.

SUPERINTENDÊNCIA DO DESENVOLVIMENTO DA AMAZÔNIA - SUDAM. Grupamento de espécies tropicais da Amazônia por similaridade de características básicas e por utilização. Belém: 1981. 237 p.

TRUGILHO, P.F. Aplicação de algumas técnicas multivariadas na avaliação da qualidade da madeira e do carvão vegetal de Eucalyptus. 1995. 160 f. Tese (Doutorado em Ciência Florestal) - Universidade Federal de Viçosa, Viçosa-MG, 1995.

VITAL, B. R. Métodos de determinação da densidade da madeira. Viçosa, MG: SIF, 1984. 21 p. (Boletim técnico, 1).

XAVIER, A. Aplicação da analise multivariada da divergência genética no melhoramento de Eucalyptus spp. 1996. 146 f. Tese (Doutorado Genética e Melhoramento de Planta) - Universidade Federal de Viçosa, 1996. 